\title{
Partially slotted silicon ring resonator covered with electro-optical polymer
}

Steglich, Patrick, Mai, Christian, Stolarek, David, Lischke, Stefan, Kupijai, Sebastian, et al.

Patrick Steglich, Christian Mai, David Stolarek, Stefan Lischke, Sebastian Kupijai, Claus Villringer, Silvio Pulwer, Friedhelm Heinrich, Joachim Bauer, Stefan Meister, Dieter Knoll, Mauro Casalboni, Sigurd Schrader, "Partially slotted silicon ring resonator covered with electro-optical polymer," Proc. SPIE 9891, Silicon Photonics and Photonic Integrated Circuits V, 98910R (13 May 2016); doi: $10.1117 / 12.2217725$

SPIE. Event: SPIE Photonics Europe, 2016, Brussels, Belgium 


\title{
Partially slotted silicon ring resonator covered with electro-optical polymer
}

\author{
Patrick Steglich ${ }^{\mathrm{a}, \mathrm{b}},{ }^{*}$, Christian Mai ${ }^{\mathrm{c}}$, David Stolarek ${ }^{\mathrm{c}}$, Stefan Lischke ${ }^{\mathrm{c}}$, Sebastian Kupijai ${ }^{\mathrm{d}}$, \\ Claus Villringer ${ }^{\mathrm{a}, \mathrm{b}}$, Silvio Pulwer ${ }^{\mathrm{a}, \mathrm{b}}$, Friedhelm Heinrich ${ }^{\mathrm{a}}$, Joachim Bauer ${ }^{\mathrm{a}}$, Stefan Meister ${ }^{\mathrm{d}}$, \\ Dieter Knoll ${ }^{c}$, Mauro Casalboni ${ }^{\mathrm{b}}$, and Sigurd Schrader ${ }^{\mathrm{a}}$ \\ aUniversity of Applied Sciences Wildau, Hochschulring 1, 15745 Wildau, Germany \\ bUniversity of Rome Tor Vergata, Via Orazio Raimondo 18, 00133 Rome, Italy \\ 'IHP, Im Technologiepark 25, 15236 Frankfurt(Oder), Germany \\ dTechnical University Berlin, Straße des 17. Juni 135, 10623 Berlin, Germany
}

\begin{abstract}
In this work, we present for the first time a partially slotted silicon ring resonator (PSRR) covered with an electro-optical polymer (Poly[(methyl methacrylate)-co-(Disperse Red 1 acrylate)]). The PSRR takes advantage of both a highly efficient vertical slot waveguide based phase shifter and a low loss strip waveguide in a single ring. The device is realized on $200 \mathrm{~mm}$ silicon-on-insulator wafers using $248 \mathrm{~nm}$ DUV lithography and covered with the electro-optic polymer in a post process. This silicon-organic hybrid ring resonator has a small footprint, high optical quality factor, and high DC device tunability. A quality factor of up to $10^{5}$ and a DC device tunability of about $700 \mathrm{pm} / \mathrm{V}$ is experimentally demonstrated in the wavelength range of $1540 \mathrm{~nm}$ to $1590 \mathrm{~nm}$. Further, we compare our results with state-of-the-art silicon-organic hybrid devices by determining the poling efficiency. It is demonstrated that the active PSRR is a promising candidate for efficient optical switches and tunable filters.
\end{abstract}

Keywords: ring resonator, silicon photonics, slot waveguides, silicon-organic hybrid

\section{INTRODUCTION}

Driven by substantial research investments the integration of silicon based photonic devices on silicon-on-insulator substrates has reached a degree of maturity that already permits industrial adoption. However, silicon has a lack of linear electro-optical (EO) coefficient and therefore linear tunable filter and modulators using advanced modulation formats like quadrature phase-shift keying (QPSK) and 16-state quadrature amplitude modulation (16QAM) are difficult to realize. ${ }^{1}$ During the last decade a different approach based on silicon slot waveguides has been proposed ${ }^{2}$ and experimentally demonstrated to be suitable as optical phase shifter. ${ }^{3,4}$ Infiltration of the interior of the slot waveguide with an EO polymer allows the use of the linear EO effect (Pockels effect).

However, slot waveguide phase shifters suffer from relatively high losses mainly caused by sidewall roughness. ${ }^{5}$ As a consequence, slot waveguide ring resonators have typically small optical quality factors (Q-factors). ${ }^{4,6,7}$ To overcome this deficiency we have recently proposed a novel ring resonator concept where the ring is only partially slotted. ${ }^{8}$ This partially slotted ring resonator (PSRR) combines an efficient slot waveguide phase shifter with a low loss and strongly guiding silicon strip waveguide in a single ring resonator. Here we demonstrate for the first time a PSRR covered by an active EO polymer to show the potential of the concept for optical tunable filters with high DC device tunability.

\footnotetext{
*E-mail: patrick.steglich@th-wildau.de
} 

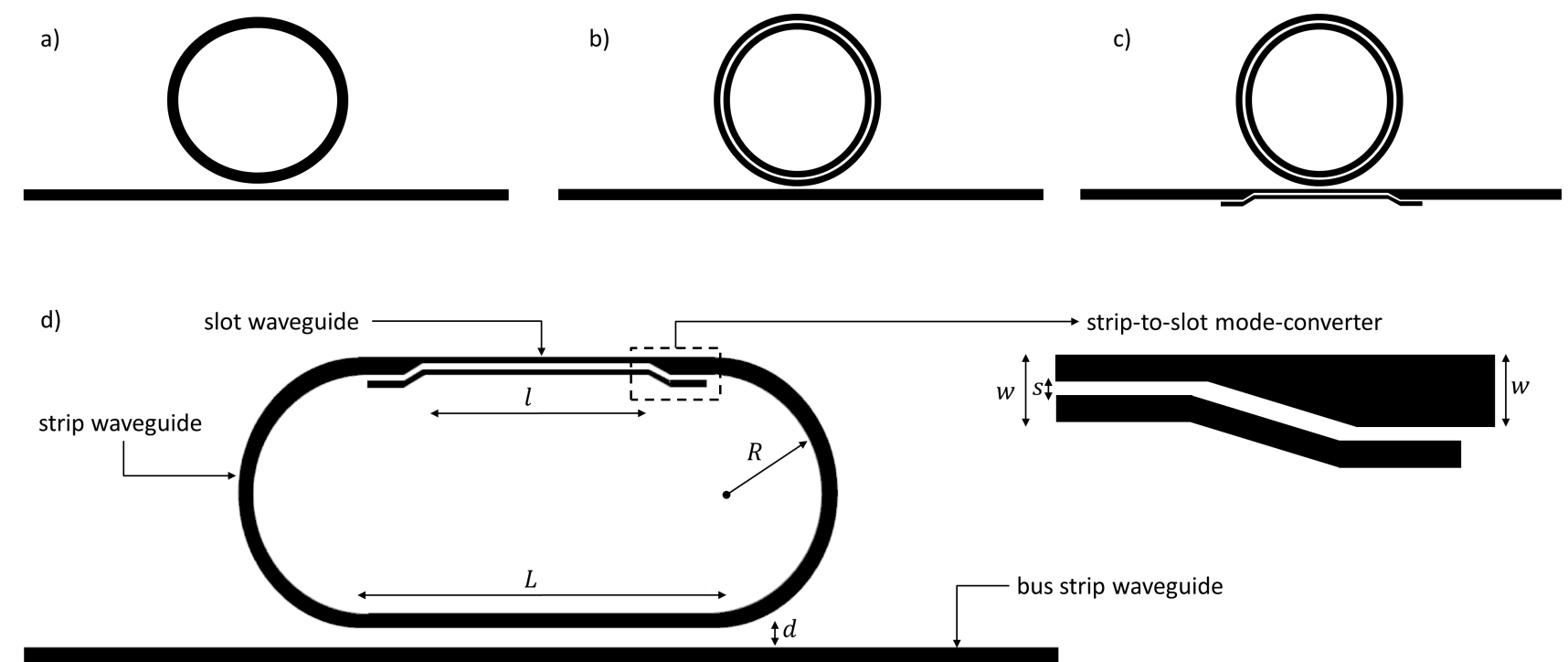

Figure 1. Schematics of different ring resonator concepts: a) Common silicon strip waveguide ring resonator. b) Fully slotted ring resonator with strip waveguide bus. c) Fully slotted ring resonator with slot waveguide bus. d) Partially slotted ring resonator with strip-to-slot mode-converter.

\section{DEVICE DESIGN AND FABRICATION}

The ring resonators were designed at University of Applied Sciences Wildau and fabricated in a $0.13 \mu \mathrm{m}$ SiGe BiCMOS pilot line at IHP in Frankfurt (Oder), Germany using $200 \mathrm{~mm}$ silicon-on-insulator wafers and $248 \mathrm{~nm}$ DUV lithography. Three ring resonators with different slot widths $(s=60 \mathrm{~nm}, 120 \mathrm{~nm}, 150 \mathrm{~nm})$ were realized. A scheme of typical ring resonator types including the partially slotted ring resonator is depicted in Figure 1. Conventional ring resonators are based on strip waveguides or slot waveguides, as depicted in Figure 1(a) and Figure 1(b), respectively. Strip-to-slot mode-converter are introduced in the bus waveguide in order to enhance the coupling efficiency in case of slot waveguide ring resonators (Figure 1(c)). In contrast to that the hybrid concept of the PSRR takes advantage of both a strongly guiding strip waveguide at the bent part and an efficient strip-loaded slot waveguide at the straight part of the oval shaped ring (racetrack configuration). A schematic of the PSRR is depicted in Figure 1(d). To exploit the advantages of each waveguide type an efficient strip-to-slot waveguide transition is needed. Therefore, we used a strip-to-slot mode-converter as depicted in Figure 1(d). The coupling distance and waveguide width are $d=200 \mathrm{~nm}$ and $w=500 \mathrm{~nm}$, respectively. The length of the strip-loaded slot waveguide is $l=12 \mu \mathrm{m}$ and the length of the strip-to-slot mode-converter is $8 \mu \mathrm{m}$. With the ring radius $R=20 \mu \mathrm{m}$ and the coupling length $L=28 \mu \mathrm{m}$ the total circumference results in $C=182 \mu \mathrm{m}$ and a small foot print of $2.72 \mu \mathrm{m}^{2}$.

A detailed cross sectional view of the strip-loaded slot waveguide structure is shown in Figure 2. The striploaded slot waveguide consists of two silicon rails with a standard silicon-on-insulator height of $h_{r}=220 \mathrm{~nm}$. All waveguides are located on top of a $2 \mu \mathrm{m}$ buried oxide layer. The silicon strip-loads were etched remaining a silicon slab height of $h_{l}=50 \mathrm{~nm}$ and additionally $n^{+}$-implanted to decrease the electrical sheet resistance. In order to avoid excessive losses due to free carrier absorption the doping concentration was reduced close to the slot waveguide structure ( $n$-implanted). The slot waveguide is electrically connected to Ground-Signal-Ground (GSG) aluminium electrodes through the doped silicon strip-loads and tungsten contacts (see Figure 2). In order to improve connectivity and conductivity a thin silicide intermediate layer was used to connect the tungsten contacts to the doped silicon to get an ohmic contact. Top view scanning-electron microscopic (SEM) images of the fabricated PSRR are shown in Figure 3. Figure 3(a) shows the GSG aluminium electrodes with a pitch of $150 \mu \mathrm{m}$ and an impedance of $50 \Omega$. Figure 3(c) shows a cross-sectional view of the slot waveguide recorded with 


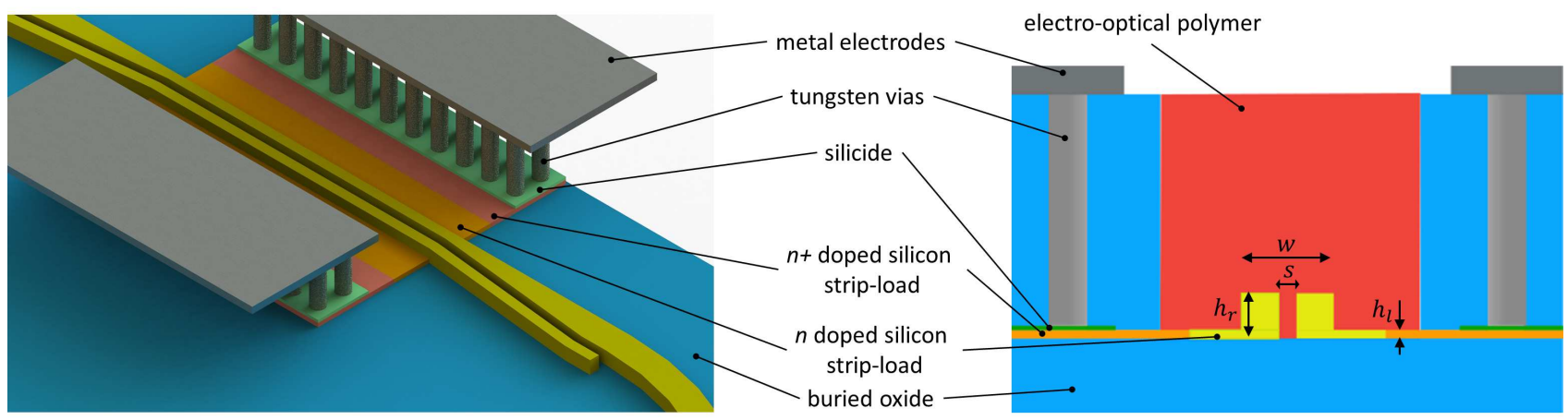

Figure 2. Detailed schematic of the strip-loaded slot waveguide part of the ring resonator and cross sectional diagram including electrical contacts (not to scale).

a focused ion beam (FIB).

A silicon dioxide top cladding is deposited and a trench is etched to open the slot waveguide structure as illustrated in Figure 2 and highlighted (blue) in Figure 3(a) and Figure 3(b). This allows the functionalization of the slot with an organic EO material. ${ }^{9}$ Using EO polymers or organic crystals as cladding material the EO effect and therefore the device tunability is expected to be significantly higher compared to free-carrier plasma dispersion based phase shifters. ${ }^{9,10}$ Further, the slot waveguide structure provides a high optical and electrical confinement which increases EO effects even more. ${ }^{11,12}$

As cladding material we have deposited a side-chain polymer system in a post-process. The polymer system consists of PMMA and the optical nonlinear chromophore Disperse Red 1 (DR1) (IUPAC Name: Poly[(methyl methacrylate)-co-(Disperse Red 1 acrylate)]). The chromophore concentration is nominally $25 \mathrm{~mol} \%$. As solvent we used 1,1,2,2-Tetrachloroethane. The resultant solution was filtered through a $0.2 \mu \mathrm{m}$ PTFE membrane filter and spin-coated on the photonic chip at $6000 \mathrm{rpm}$. To ensure that the solvent was removed after spin-coating the photonic chips were heated on a hot plate in $10{ }^{\circ} \mathrm{C}$ steps from $30{ }^{\circ} \mathrm{C}$ to $60{ }^{\circ} \mathrm{C}$ for $2 \mathrm{~h}$ each step. This was followed by heating the chips to $80^{\circ} \mathrm{C}$ for $10 \mathrm{~h}$ in a vacuum oven.

\section{DEVICE CHARACTERIZATION}

The transmission spectra of the fabricated device were obtained by a testing platform using a tunable external cavity laser (ECL) as light source with a bandwidth of $<1 \mathrm{pm}$ and maximum output power of $14 \mathrm{dBm}$. The tuning range was between $1540 \mathrm{~nm}$ and $1580 \mathrm{~nm}$ in $1 \mathrm{pm}$ steps. We used the ECL and a photodiode to measure the optical quality factor. For active measurements we employed a superluminescent diode as light source with a maximum output power of $10 \mathrm{dBm}$ and an optical spectrum analyzer to obtain the transmission spectra. In both cases the light was coupled into the silicon waveguides through a fiber grating coupler and out-coupled through another grating coupler. Due to the polarization dependency of the grating coupler the polarization of the input light was controlled using a paddle style fiber polarization rotator in order to achieve TE-polarization. The photonic chip was measured on a temperature-controlled sample holder and stabilized to $35{ }^{\circ} \mathrm{C}$ using a hot plate in order to avoid changes in the transmission due to temperature fluctuation. For active measurements the GSG electrodes are connected to an electric power source through tungsten DC probes.

\section{RESULTS AND DISCUSSION}

\subsection{DC device tunability}

The DC device tunability was obtained by measuring the transmission at several DC voltages in a non-sequential fashion in order to exclude thermal drifts as the cause of the peak shifting behavior. An approximately linear wavelength shift was measured in the voltage range of $3 \mathrm{~V}$ to $7 \mathrm{~V}$, as shown in Figure 4(a). The highest DC 

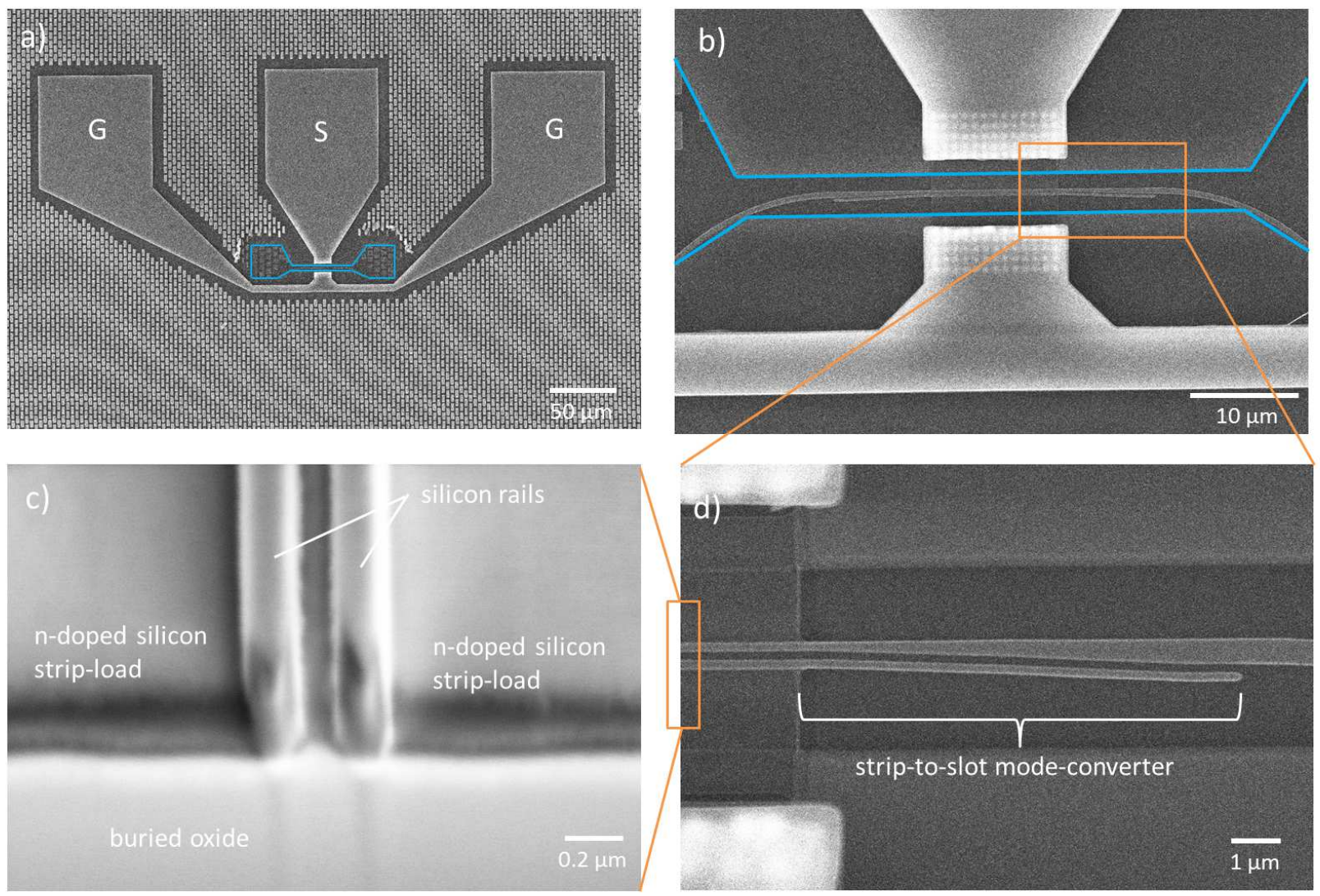

Figure 3. a) SEM image of the Ground-Signal-Ground (GSG) aluminium electrode from the top view with a trench (highlighted in blue) to functionalize the slot waveguide by an organic cladding material. b) SEM image of part of the ring resonator with strip-to-slot mode-converter and strip-loaded slot waveguide. c) FIB image of the strip-loaded slot waveguide. d) Magnification of the strip-to-slot mode-converter for the $150 \mathrm{~nm}$ slot waveguide.

device tunability of $707 \mathrm{pm} / \mathrm{V}$ has been achieved with the $110 \mathrm{~nm}$ slotted ring. With the narrow full width at half maximum of $15 \mathrm{pm}$ and a corresponding optical quality factor of up to $10^{5}$, as presented in our previous work, ${ }^{8}$ this appears to be a convenient tuning range for most applications and offers the possibility for efficient on-off keying. Further, it should be highlighted that the achieved DC device tunability is more than 20 times higher compared to common ring-based modulators making use of the plasma dispersion effect. ${ }^{13}$

\subsection{Poling efficiency}

In order to compare our results with state-of-the-art silicon-organic hybrid devices we determined the poling efficiency which is the ratio between the linear EO coefficient and the applied poling field, $r_{33} / E_{p o l} .{ }^{9}$ The poling field is given by $E_{p o l}=U / s$, where $U$ is the applied voltage. With a linear regression we determined a poling efficiency of $0.31 \mathrm{~nm}^{2} / \mathrm{V}^{2}$ for the $110 \mathrm{~nm}$ and $150 \mathrm{~nm}$ slot waveguides as depicted in Figure 4(b). A poling efficiency of $0.09 \mathrm{~nm}^{2} / \mathrm{V}^{2}$ was obtained for the $60 \mathrm{~nm}$ slot waveguide. This small poling efficiency can be explained by a non-homogeneous infiltration of the narrow slot. However, our results are in good agreement with other publications were a guest-host polymer system consisting of PMMA and YLD124 was used and poling efficiency of $0.23 \mathrm{~nm}^{2} / \mathrm{V}^{2}$ is reported. ${ }^{9}$ It should be mentioned that even higher poling efficiencies of about 

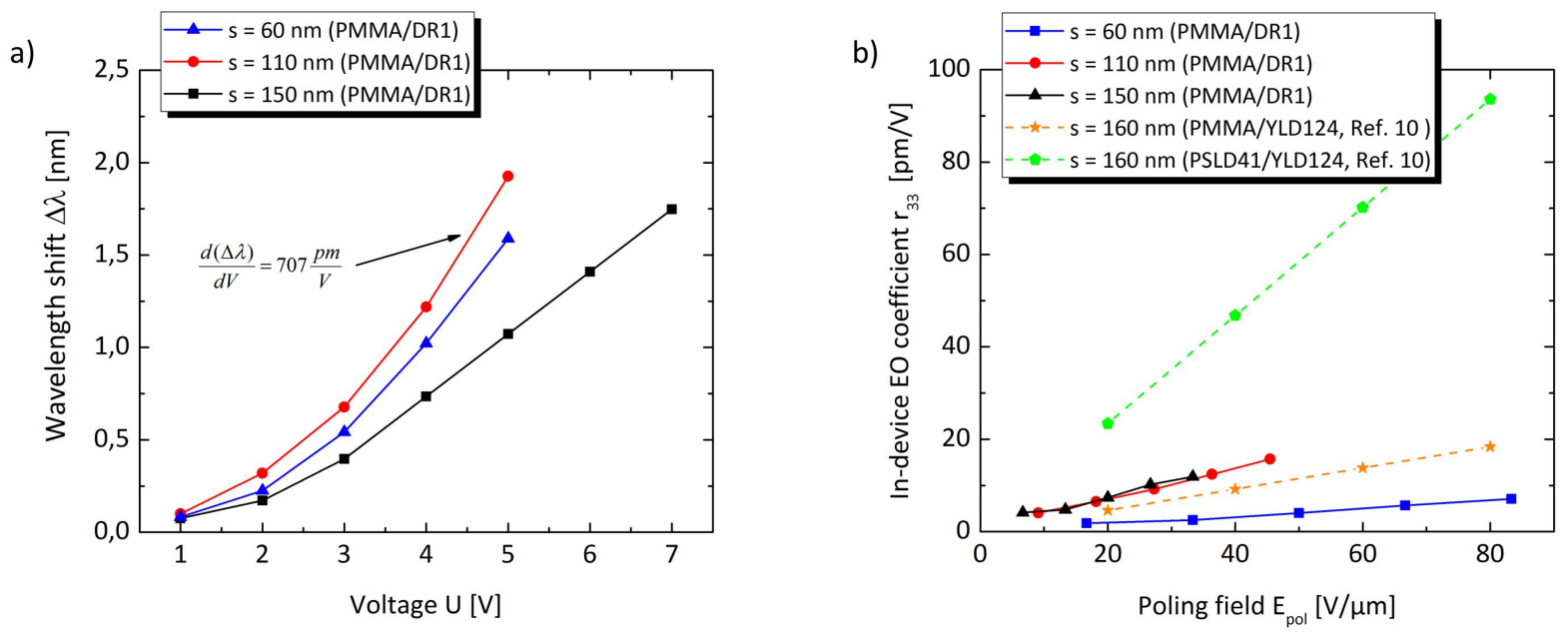

Figure 4. a) Voltage induced peak shift of the PSRR covered with PMMA/DR1. A maximum DC device tunability of $707 \mathrm{pm} / \mathrm{V}$ is obtained from the PSRR with a slot width of $110 \mathrm{~nm}$. b) Linear EO coefficient as function of applied electric field for different slot widths $(s)$. The slope of this lines are used to determine the poling efficiencies.

$0.92 \mathrm{~nm}^{2} / \mathrm{V}^{2}$ has been demonstrated recently with PSLD41 doped by $25 \mathrm{~mol} \%$ YLD124 (binary chromophore organic glass). ${ }^{9}$

\subsection{In-device linear EO coefficient}

The most limiting factor of the presented PSRR is the EO polymer. We derive the in-device EO coefficient $r_{33}$ assuming a phase shift $\Delta \phi=\Delta n \cdot k_{0} \cdot l \cdot \Gamma_{\text {slot }}$ induced by the refractive index change $\Delta n=1 / 2 \cdot n_{\text {poly }}^{3} \cdot r_{33} \cdot E$. From the experimentally obtained wavelength shift the in in-device EO coefficient can be determined by

$$
r_{33}=\frac{2 \cdot s \cdot \Delta \lambda}{n_{p o l y}^{3} \cdot l \cdot U \cdot \Gamma_{\text {slot }}}
$$

where $n_{\text {poly }}$ is the refractive index of the EO polymer and $\Gamma_{\text {slot }}$ is the field confinement factor of the slot region. ${ }^{14}$ The field confinement factor is usually defined as the ratio of the time averaged power flow in the slot area over the time averaged power flow in the total area. ${ }^{15}$ Please note that in case of silicon-on-insulator slot waveguides a significant difference between the field confinement factor for the slot region and the whole cladding region exists. ${ }^{12}$ The field confinement factor in the cladding region is about four times greater than in the slot region. ${ }^{11}$ Here we use the slot region because the linear EO effect needs a non-centrosymmetrical orientation of the EO polymer. This is only guaranteed inside the slot because of the homogeneous electric field. For the slot width of $110 \mathrm{~nm}$ and a wavelength of $\lambda=1550 \mathrm{~nm}$ the field confinement factor is $\Gamma_{\text {slot }} \approx 0.2{ }^{11}$ The indevice $\mathrm{EO}$ coefficient of the side-chain polymer system is determined to be $r_{33}=12.8 \mathrm{pm} / \mathrm{V}$ assuming a voltage of $U=1 \mathrm{~V}$ applied to the $110 \mathrm{~nm}$ wide slot waveguide with a wavelength shift of $707 \mathrm{pm}$. For comparison, the aforementioned binary chromophore organic glass (PSLD41/YLD124) exhibits a record-high in-device EO coefficient of $r_{33}=230 \mathrm{pm} / \mathrm{V} .{ }^{16}$ Using such advanced EO polymers the performance of the PSRR can be significantly improved.

\section{CONCLUSION}

In conclusion, we have demonstrated a PSRR covered with an EO polymer enabling the use of slot waveguide phase shifter while maintaining a high Q-factor and a small footprint. Under carefully controlled conditions we have experimentally shown a remarkable DC device tunability of $707 \mathrm{pm} / \mathrm{V}$ and a poling efficiency of 
$0.31 \mathrm{~nm}^{2} / \mathrm{V}^{2}$ using a commercial side-chain Polymer (PMMA/DR1) with a moderate in-device EO coefficient of $12.8 \mathrm{pm} / \mathrm{V}$. The PSRR appears to be promising for various fields of applications for integrated opto-electronics. In particular, the proposed design will be useful for integrated silicon-organic hybrid photonic devices such as optical switches, modulators, and tunable filters.

\section{ACKNOWLEDGMENTS}

We gratefully acknowledge the financial support provided by the German Federal Ministry of Education and Research (BMBF) under contract no. 03FH086PX2. We additionally thank P. Prosposito, F. De Matteis, and R. De Angelis from the University of Rome Tor Vergata, Italy, and A. Al-Saadi from the Technical University Berlin, Germany, for their encouragement. Furthermore, we thank J. Katzer, H. Silz, L. Zimmermann, B. Tillack, and A. Mai from the Institute of High-Performance Microelectronics (IHP), Germany for their support in the framework of the Joint-Lab IHP/TH Wildau.

\section{REFERENCES}

[1] Lauermann, M., Palmer, R., Koeber, S., Schindler, P. C., Korn, D., Wahlbrink, T., Bolten, J., Waldow, M., Elder, D. L., Dalton, L. R., Leuthold, J., Freude, W., and Koos, C., "Low-power silicon-organic hybrid (soh) modulators for advanced modulation formats," Opt. Express 22, 29927-29936 (Dec 2014).

[2] Almeida, V. R., Xu, Q., Barrios, C. A., and Lipson, M., "Guiding and confining light in void nanostructure," Opt. Lett. 29, 1209-1211 (Jun 2004).

[3] Leuthold, J., Koos, C., Freude, W., Alloatti, L., Palmer, R., Korn, D., Pfeifle, J., Lauermann, M., Dinu, R., Wehrli, S., Jazbinsek, M., Gunter, P., Waldow, M., Wahlbrink, T., Bolten, J., Kurz, H., Fournier, M., Fedeli, J.-M., Yu, H., and Bogaerts, W., "Silicon-organic hybrid electro-optical devices," Selected Topics in Quantum Electronics, IEEE Journal of 19, 114-126 (Nov 2013).

[4] Baehr-Jones, T., Hochberg, M., Wang, G., Lawson, R., Liao, Y., Sullivan, P., Dalton, L., Jen, A., and Scherer, A., "Optical modulation and detection in slotted silicon waveguides," Opt. Express 13, 5216-5226 (Jul 2005).

[5] Ding, R., Baehr-Jones, T., Kim, W.-J., Xiong, X., Bojko, R., Fedeli, J.-M., Fournier, M., and Hochberg, M., "Low-loss strip-loaded slot waveguides in silicon-on-insulator," Opt. Express 18, 25061-25067 (Nov 2010).

[6] Gould, M., Baehr-Jones, T., Ding, R., Huang, S., Luo, J., Jen, A. K.-Y., Fedeli, J.-M., Fournier, M., and Hochberg, M., "Silicon-polymer hybrid slot waveguide ring-resonator modulator," Opt. Express 19, 3952-3961 (Feb 2011).

[7] Claes, T., Molera, J., De Vos, K., Schachtb, E., Baets, R., and Bienstman, P., "Label-free biosensing with a slot-waveguide-based ring resonator in silicon on insulator," Photonics Journal, IEEE 1, 197-204 (Sept 2009).

[8] Steglich, P., Mai, C., Stolarek, D., Lischke, S., Kupijai, S., Villringer, C., Pulwer, S., Heinrich, F., Bauer, J., Meister, S., Knoll, D., Casalboni, M., and Schrader, S., "Novel ring resonator combining strong field confinement with high optical quality factor," Photonics Technology Letters, IEEE 27, 2197-2200 (Oct 2015).

[9] Palmer, R., Koeber, S., Elder, D. L., Woessner, M., Heni, W., Korn, D., Lauermann, M., Bogaerts, W., Dalton, L., Freude, W., Leuthold, J., and Koos, C., "High-speed, low drive-voltage silicon-organic hybrid modulator based on a binary-chromophore electro-optic material," Journal of Lightwave Technology 32, 2726-2734 (Aug 2014).

[10] Korn, D., Jazbinsek, M., Palmer, R., Baier, M., Alloatti, L., Yu, H., Bogaerts, W., Lepage, G., Verheyen, P., Absil, P., Guenter, P., Koos, C., Freude, W., and Leuthold, J., "Electro-optic organic crystal silicon high-speed modulator," Photonics Journal, IEEE 6, 1-9 (April 2014).

[11] Steglich, P., Villringer, C., Dümecke, S., Michel, Y. P., Casalboni, M., and Schrader, S., "Silicon-on-insulator slot-waveguide design trade-offs," in [Proceedings of the 3rd International Conference on Photonics, Optics and Laser Technology], 47-52 (2015). 
[12] Steglich, P., Villringer, C., Dümecke, S., Michel, Y. P., Casalboni, M., and Schrader, S., "Design optimization of slot-waveguides covered with organic cladding materials for integrated photonic devices," in $[N W K$ 16], Middendorf, S. and Hüttinger, G., eds., 192-198, BERLINER WISSENSCHAFTS-VERLAG GmbH (4 2015).

[13] Xuan, Z., Ma, Y., Liu, Y., Ding, R., Li, Y., Ophir, N., Lim, A. E.-J., Lo, G.-Q., Magill, P., Bergman, K., Baehr-Jones, T., and Hochberg, M., "Silicon microring modulator for $40 \mathrm{gb} / \mathrm{s}$ nrz-ook metro networks in o-band," Opt. Express 22, 28284-28291 (Nov 2014).

[14] Chuang, S. L., [Physics of Photonic Devices], Wiley, 2 ed. (1 2009).

[15] Robinson, J. T., Preston, K., Painter, O., and Lipson, M., "First-principle derivation of gain in high-indexcontrast waveguides," Opt. Express 16, 16659-16669 (Oct 2008).

[16] Palmer, R., Koeber, S., Woessner, M., Elder, D. L., Heni, W., Korn, D., Yu, H., Lauermann, M., Bogaerts, W., Dalton, L. R., Freude, W., Leuthold, J., and Koos, C., "High-speed silicon-organic hybrid (soh) modulators with $230 \mathrm{pm} / \mathrm{v}$ electro-optic coefficient using advanced materials," in [Optical Fiber Communication Conference], Optical Fiber Communication Conference, M3G.4, Optical Society of America (2014). 